\title{
LA RESPONSABILIDAD SOCIAL CORPORATIVA EN LAS EMPRESAS AGROALIMENTARIAS: UN ANÁLISIS DE SU DIVULGACIÓN MEDIANTE INFORMES DE SOSTENIBILIDAD
}

\author{
Carlos Anguiano-Santos ${ }^{a *}$, Mercedes Luque-Vílchez $^{a}$, Melania Salazar-Ordóñez $^{a}$
}

\author{
a WEARE-Water, Environmental, and Agricultural Resources Economics, Dpto. Economía Agraria, \\ Finanzas y Contabilidad, Universidad de Córdoba (Córdoba, d42ansac@uco.es, mercedes.luque@uco.es, \\ msalazar@uco.es)
}

\begin{abstract}
Resumen
Las empresas agroalimentarias son consideradas industrias medioambientalmente sensibles, adquiriendo cada vez más relevancia la divulgación de información sobre el impacto de sus actividades en términos de sostenibilidad. Este estudio analiza el nivel de divulgación de la información sobre sostenibilidad divulgada por empresas del sector agroalimentario español. Para ello, se realiza un análisis de contenido de informes de sostenibilidad publicados por empresas pertenecientes al sector agroalimentario de acuerdo con los estándares exigidos tras la implementación de la Ley 11/2018, transposición española de la Directiva 2014/95/EU, es decir, en los años 2018 o 2019. Los resultados indican que las empresas tienen dificultades (o poco interés) en divulgar información sobre Bloques como el de Medioambiente, Derechos Humanos, y Anticorrupción y Soborno. Además, se obtienen mejores resultados en el nivel de divulgación para aquellos Bloques que representan información más laxa, como la incluida en Comunidades Locales Sociales, y Empleados. Esto lleva a cuestionar el nivel de divulgación del sector agroalimentario español, pese a la elevada la influencia, en la conformación de las preferencias ciudadanas, del conocimiento sobre prácticas sostenibles. Así, las empresas deben comenzar a preocuparse no solo por divulgar, como exige la mencionada Directiva, sino también porque la información divulgada sea completa y de calidad.
\end{abstract}

Palabras clave: Informes de sostenibilidad, Global Reporting Initiative (GRI), Directiva 95/2014, Ley 11/2018, Sector agroalimentario.

\section{Introducción}

El sector agroalimentario es una industria ambiental y socialmente sensible [Cajamar (2019)], particularmente en España donde además resulta fundamental en términos económicos [Ministerio de Agricultura, Pesca y Alimentación (2018); Comisión Europea (2019)], convirtiéndose a lo largo del tiempo en una preocupación clave no solo para los gobiernos sino también para la sociedad en general [Freeman (1984); Gray et al. (1995)]. Una importante fuente de información sobre el desempeño ambiental y social de las empresas, que puede ayudar a su monitoreo, son los denominados informes de sostenibilidad. No obstante, existen autores que afirman que, pese a su relevancia, la información de sostenibilidad vertida en dichos informes no consigue transmitir una imagen fidedigna de la actividad desarrollada por las empresas [Baviera-Puig et al. (2014); Luhmann y Theuvsen (2016); Luque-Vílchez y Larrinaga (2016)], siendo para las empresas agroalimentarias el número de informes completos publicados y el nivel de información contenido en los mismos bajo [Sodano y Hingley (2018)]. En este contexto, este estudio pretende medir el nivel de divulgación de los informes de sostenibilidad, analizando la información divulgada por 23 empresas agroalimentarias españolas, mediante un análisis de contenido basado en la guía GRI (Global Reporting Initiative) [GRI (2017)].

\section{Metodología}

Para llevar a cabo el análisis, se utilizó una muestra que se correspondió con el total de empresas del sector agroalimentario que habían presentado informes de sostenibilidad siguiendo las directrices de Global Reporting Initiative (GRI) entre los años 2018 y 2019. Estos años fueron elegidos con el objeto de medir el nivel de divulgación de la información reportada por las empresas después de la entrada en vigor de la Ley 11/2018 [Gobierno de España (2018)], transposición de la Directiva 2014/95/UE [Comisión Europea (2014)] que hacía obligatorio el reporte a empresas y grupos con más de 500 trabajadores o que alcanzaran determinados volúmenes de activos, cifra de negocio y/o contaran con más de 250 empleados. Así, se encontraron un total de 23 empresas. Una vez obtenida la información, se llevó a cabo un análisis de contenido, codificando información cualitativa para obtener escalas cuantitativas, considerando la guía de Global Reporting Initiative [GRI (2016)]. Por tanto, se recabó la existencia de información en 5 Bloques conformados por 19 Sub-bloques, lo que supuso el análisis de un total 60 indicadores.

\section{Resultados}

Los resultados obtenidos mostraron la existencia de una gran heterogeneidad entre la información de sostenibilidad divulgada por las empresas en cada Bloque, aunque en ninguno de ellos se alcanzó el 50\% de información divulgada. Los Bloques de Comunidades Sociales Locales y Empleados presentaron el 
mayor nivel de divulgación con el 48\% y 44,6\%, respectivamente. Mientras el Bloque Anticorrupción y soborno mostró el nivel más bajo de divulgación. Por lo que, el nivel medio en la divulgación de información de sostenibilidad obtenido fue de un 35,3\% (Gráfico 1).

Gráfico 1: Índice de divulgación global

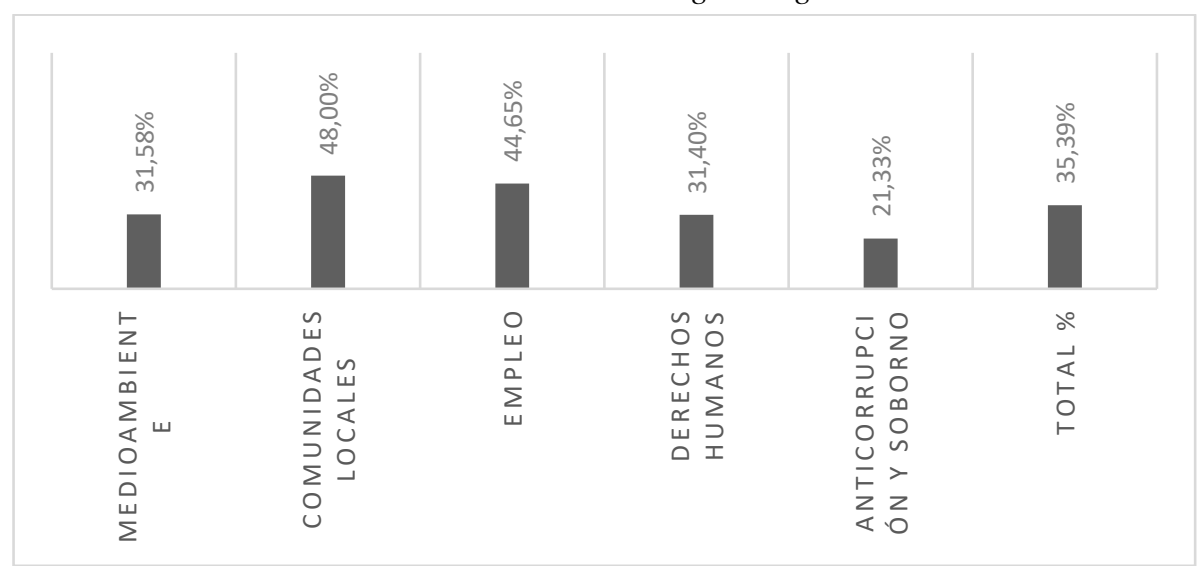

A continuación, se presenta un análisis detallado del nivel de divulgación de cada Bloque, mostrando sus correspondientes Sub-bloques, comenzando con el Gráfico 2, donde aparece el Bloque Medioambiente.

Gráfico 2: Índice de divulgación del Bloque Medioambiente

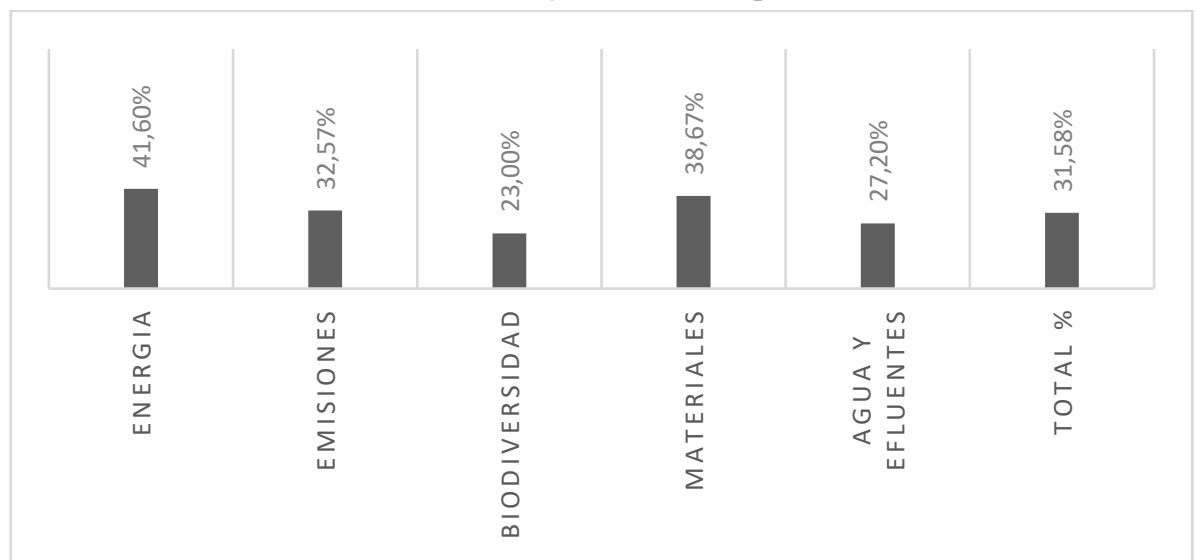

El mayor nivel de información divulgada, aunque de nuevo sin alcanzar el 50\%, se encontró en los Subbloques de Energía y Materiales. Por el contrario, solo el 23\% de la información de Biodiversidad fue reportada. Cabe mencionar que la mayoría de las empresas (88\%) informaron sobre el consumo eléctrico real (indicador 302-1 del Bloque Energía).

En cuanto al Bloque de Comunidades Locales Sociales, la Gráfica 3, al igual que la previa, muestra el nivel de divulgación en el único Sub-bloque que la conforma, estando además compuesto por solo dos indicadores, siendo la información de sostenibilidad menos representada y medida por los Estándares GRI (GRI, 2016).

Gráfico 3: índice divulgación del Bloque Comunidades locales sociales

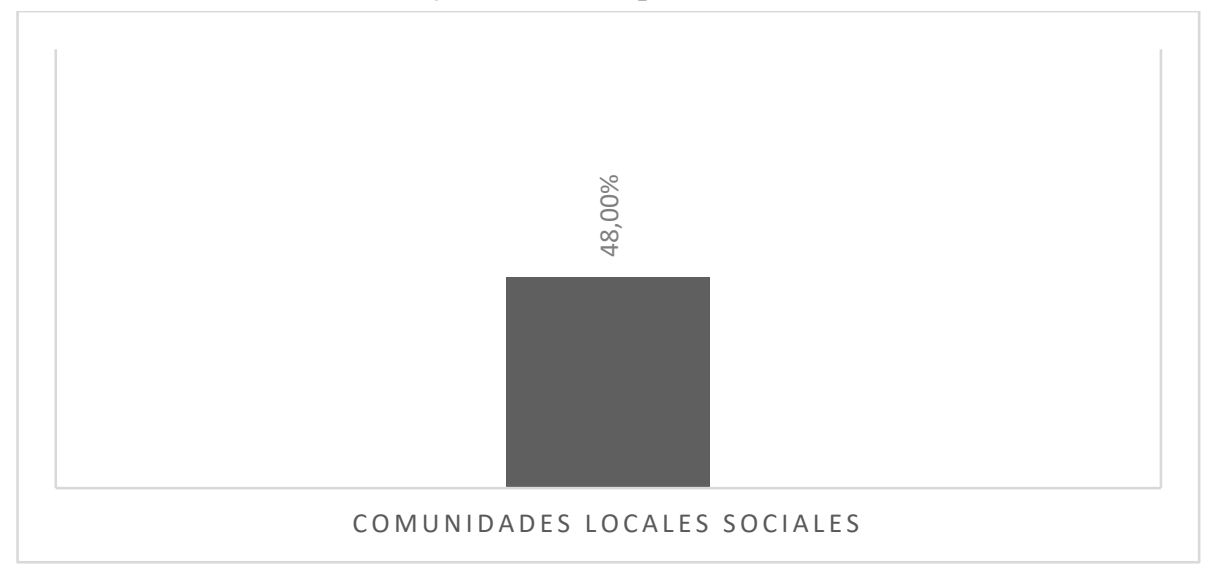


El nivel general de divulgación del Sub-bloque fue, obviamente al igual que para el Bloque, del 48\%, dado por un indicador con un nivel de reporte superior al 50\% que midió las actividades de las empresas que involucran a las comunidades locales (413-1) y otro por debajo del $50 \%$ que indicó las operaciones con impactos negativos significativos reales y potenciales en las comunidades locales (413-2).

Los resultados desagregados por Sub-bloques de Empleados se presentan en la siguiente Gráfico 3, se debe indicar que el Sub-bloque de Salud y Seguridad en el trabajo cuenta de 10 indicadores de los 19 que integran la totalidad del Bloque.

Gráfico 3: índice divulgación del Bloque Empleo

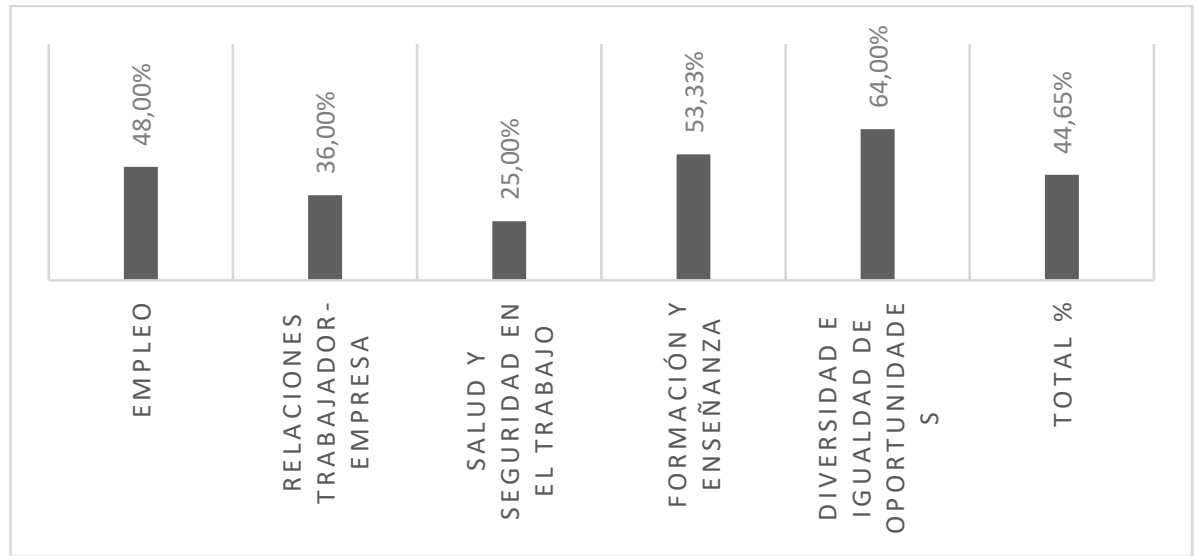

Este Bloque de información mostró tres Sub-bloques con buen nivel de divulgación en comparación con algunos de los Bloque analizados previamente: Empleo, Formación y Enseñanza, y Diversidad e Igualdad de Oportunidades, con unos niveles de $48 \%, 53,3 \%$ y $64 \%$ respectivamente, apareciendo por primera vez niveles de divulgación por encima del 50\%. No obstante, la información contenida en estos Sub-bloques tenía una naturaleza administrativa pudiéndose recolectar con más facilidad.

El cuarto Bloque de información sobre sostenibilidad, Derechos Humanos, se describe a continuación (Gráfico 4). Contiene el mayor número de Sub-bloques (8) pero descrito por datos limitados, dado que seis Sub-bloques están representados por un solo indicador.

Gráfico 4: Índice divulgación del Bloque Derechos humanos

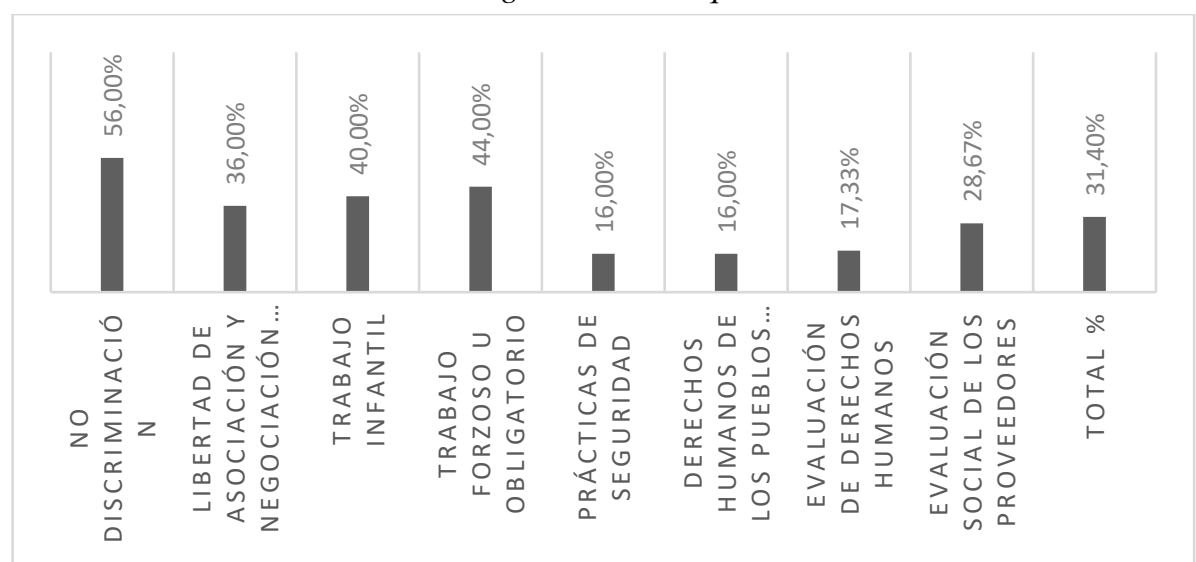

Solo uno de los ocho Sub-bloques reveló un nivel de divulgación de información superior al 50\%, el denominado No Discriminación, aunque el Sub-bloque de Trabajo Forzoso u Obligatorio se situó cerca de este porcentaje, seguido del Sub-bloque Libertad de Asociación y Negociación. No obstante, dicho Bloque sobre No discriminación aparecía formado por un solo indicador (406-1). Añadir que se pudo observar como las empresas parecían tener dificultades para divulgar información relativa a los Sub-bloques Prácticas de Seguridad, Derechos Humanos de los pueblos indígenas y Evaluación de Derechos Humanos. Finalmente, la información de sostenibilidad relacionada con el Bloque Anticorrupción y Soborno se describe en la Gráfico 5. Se descompone en tres Sub-bloques y solo cuenta con cuatro indicadores. 
Gráfico 5: Índice divulgación del Bloque Anticorrupción y soborno

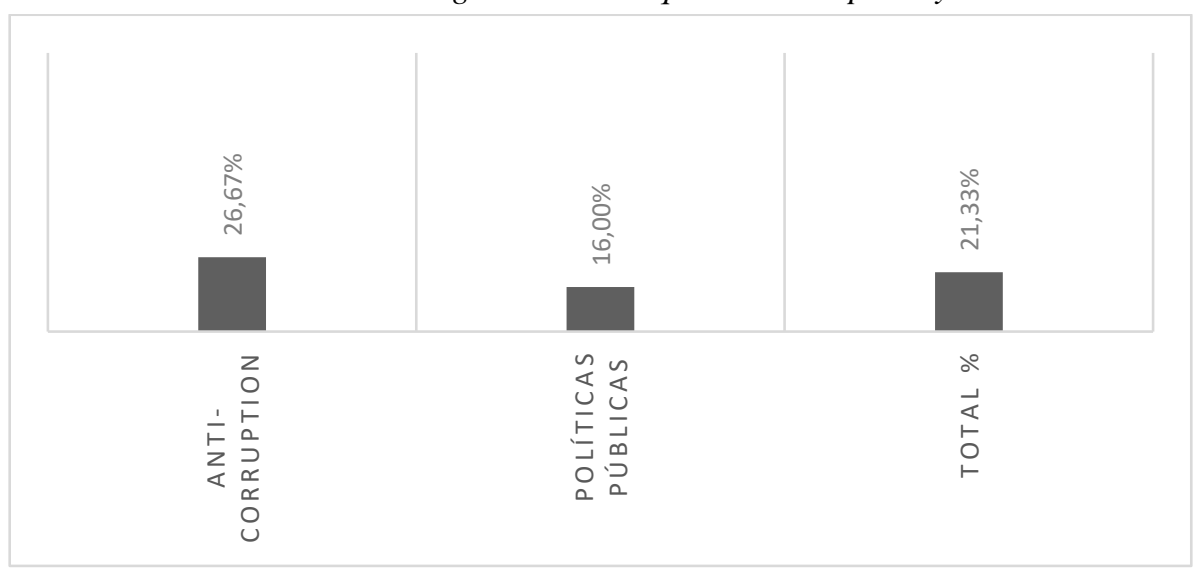

Este bloque de información de sostenibilidad no presentó Sub-bloques con un gran nivel de divulgación, ya que el Sub-bloque con más información reportada fue Anti-Corrupción con un 26,67\%, dentro de este el indicador más divulgado fue el relacionado con la comunicación y capacitación sobre políticas y procedimientos de anticorrupción (205-2).

\section{Conclusiones}

Los resultados muestran que no se realizan esfuerzos suficientes para fomentar la divulgación de información de sostenibilidad en el sector agroalimentario. A pesar de que el número de informes de sostenibilidad publicados en el sector agroalimentario a nivel mundial ha aumentado con el tiempo, esta actividad aún se encuentra en una etapa embrionaria, en general, y, particularmente, cuando se compara con otros sectores como por ejemplo el financiero. Si bien existe presión desde distintos ámbitos de la sociedad por la elaboración y divulgación de información de sostenibilidad en relación con la producción de alimentos, las empresas analizadas aún no parecen haber interiorizado la relevancia de realizar informes de sostenibilidad que ofrezcan transparencia en el comportamiento empresarial.

\section{Bibliografia}

Baldini, M., Dal Maso, L., Liberatore, G., Mazzi, F., Tezani, S., 2018. Role of country- and firm-level determinants in environmental. Journal of Business Ethics, 150(1): 79-98.

Baviera-Puig, A., García-Martínez, G., \& Gómez-Navarro, T. (2014). Propuesta metodológica mediante ANP para la evaluación de memorias de sostenibilidad del sector agroalimentario español. Economía Agraria y Recursos Naturales, 14(1): 81-101.

Cajamar, 2019. Observatorio sobre el sector agroalimentario español en el contexto europeo, Universidad de Valencia, Valencia.

Comisión Europea, 2014. Non-financial reporting EU rules require large companies to publish regular reports on the social and environmental impacts of their activities.

Comisión Europea, 2019. Communication from the Commission to the European Parliament, the European Council, the European Economic and Social Committee and the Committee of the regions. The European Green Deal. Brussels.

Freeman, E. R. (1984). Strategic Management: A Stakeholder Approach. Cambridge: Pitman Publishing.

Gray, R., Kouhy, R., \& Lavers, S. (1995). Corporate social and environmental reporting: a review of the literature and a longitudinal study of UK disclosure. Accounting, Auditing \& Accountability Journal, 8(2): 47-77.

Gobierno de España (2018). Ley 11/2018, de 28 de diciembre. BOE, número 314, 29 de diciembre. Madrid.

GRI., 2016. GRI Standard. https://www.globalreporting.org/standards

GRI., 2017. Linking the GRI Standards and the European Directive on non-financial and diversity disclosure.

Luhmann, H., \& Theuvsen, L. (2016). Corporate social responsibility in agribusiness: Literature review and future research directions. Journal of Agricultural and Environmental Ethics, 4(29), 673-696.

Luque Vílchez, M., \& Larrinaga, C. (2016). Reporting Models do not Translate Well: Failing to Regulate CSR Reporting in Spain. Social and Environmental Accountability Journal, 36(1): 56-75.

Ministerio de Agricultura, Pesca y Alimentación (2018a). Informe anual de la industria alimentaria española periodo 2017-2018. Madrid: Dirección General de la Industria Alimentaria.

Sodano, V., Hingley, M.K., 2018. Corporate Social Responsibility reporting: the case of the agri-food sector. Economia Agro-Alimentare/Food Economy, 20(1): 93-119. 